\title{
Downregulation of SOX3 leads to the inhibition of the proliferation, migration and invasion of osteosarcoma cells
}

\author{
YANJIE GUO*, JIMIN YIN*, MINGJIE TANG* and XINGANG YU \\ Department of Orthopedic Surgery, Shanghai Sixth People's Hospital, \\ Shanghai Jiao Tong University, Shanghai 200233, P.R. China
}

Received August 30, 2016; Accepted January 24, 2018

DOI: $10.3892 /$ ijo.2018.4278

\begin{abstract}
Sex determining region Y-box protein 3 (SOX3) is involved in embryonic development and tumorigenesis. However, the expression and precise role of SOX3 in osteosarcoma remain unclear. In this study, we reported that SOX3 expression was upregulated in osteosarcoma tissues compared with non-cancerous bone cyst tissues. To elucidate the cellular and molecular function of SOX3, we examined the consequences of SOX3 knockdown in osteosarcoma cells. We found that the downregulation of SOX3 inhibited the proliferation, migration and invasion of osteosarcoma cells. SOX3 downregulation also increased the cell population in the G1 phase and induced cell apoptosis. SOX3 knockdownmediated cell cycle arrest and cell apoptosis were associated with decreased levels of Cdc25A, cyclin D1, proliferating cell nuclear antigen (PCNA) and Bcl-2, as well as an increased Bax expression. We also found that the downregulation of SOX3 decreased the expression of Snail, Twist and matrix metalloproteinase-9 (MMP-9), and increased E-cadherin expression, resulting in the inhibition of cell migration and invasion. Taken together, our data indicate that SOX3 may serve as an oncogene in osteosarcoma, and SOX3 downregulation may prove to be a novel approach for the inhibition of osteosarcoma progression.
\end{abstract}

\section{Introduction}

Osteosarcoma is the most common primary malignant bone tumor arising from long bones in children and adolescents (1). During the past decades, treatment strategies combining surgery, chemotherapy and in some cases radiotherapy, have increased the 5-year survival rates of patients with localized tumor from $20 \%$ to approximately $60 \%$ (2). However, the

Correspondence to: Dr Xingang Yu, Department of Orthopedic Surgery, Shanghai Sixth People's Hospital, Shanghai Jiao Tong University, 600 Yishan Road, Shanghai 200233, P.R. China

E-mail:xgxuxh@163.com

*Contributed equally

Key words: sex determining region Y-box protein 3, osteosarcoma, cell proliferation, migration, invasion prognosis of patients with advanced osteosarcoma remains unfavorable due to recurrence, distant metastasis and resistance to treatment (3). Therefore, novel diagnostic biomarkers and therapeutic targets for patients with osteosarcoma are required.

Sex determining region Y-box protein (SOX)3 is a member of SOX family transcription factors, which selectively interact with the common target sequence (A/T)ACAA(A/T)G and activate gene transcription. SOX3, together with SOX1, SOX2 and SOX21, belongs to the subgroup of the SOX B family (4). SOX3 is believed to play a critical role during embryonic development (5-7). In recent years, evidence has been provided to suggest the involvement of SOX3 in tumorigenesis. Xia et al reported that SOX3 was able to induce the oncogenic transformation of chicken embryo fibroblasts (8). Yang et al and Cai et al indicated that SOX3 acted as an oncogene in human epithelial ovarian cancer (9) and esophageal squamous cell carcinoma (ESCC) (10). Although other members of the SOX family, including SOX2 (11,12), SOX9 (13) and SOX18 (14), have been found to be involved in the development of osteosarcoma, the expression and biological function of SOX3 in osteosarcoma remain unclear.

In this study, we demonstrated that SOX3 was upregulated in osteosarcoma tissues in comparison with non-cancerous bone cyst tissues. To determine whether SOX3 is involved in the development and progression of osteosarcoma, we carried out the functional characterization of SOX3 in human osteosarcoma cell lines in which SOX3 was silenced. We further investigated the mechanisms underlying the effects of SOX3 knockdown on osteosarcoma. Our results indicate that SOX3 may act as an oncogene in osteosarcoma by regulating cell proliferation, migration and invasion.

\section{Materials and methods}

Patients and tumor sample preparations. This study was approved by the Ethics Committees of Shanghai Sixth People's Hospital, Shanghai, China. Written consent was obtained from all the enrolled patients for the use of tissue specimens. A total of 70 patients with primary osteosarcoma and 20 patients with bone cysts admitted to Department of Orthopedic Surgery, Shanghai Sixth People's Hospital were enrolled in this study. All collected primary osteosarcoma and non-cancerous bone cyst tissue samples were immediately frozen in liquid nitrogen and stored at $-80^{\circ} \mathrm{C}$ until use. 
RNA extraction and RT-qPCR. TRIzol (Invitrogen, Carlsbad, CA, USA) was used to extract total RNA according to the manufacturer's instructions. Total RNA was then reverse transcribed with Moloney murine leukemia virus reverse transcriptase (M-MLV RT; Promega, Madison, WI, USA). Quantitative (real-time) PCR was carried out on an ABI 7300 real-time PCR machine (Applied Biosystems, Foster City, CA, USA). The sequences of the primers used were as follows: SOX3, forward, 5'-TGAACTCAAGAACCCCGTAGG-3' and reverse, 5'-GCTGCGTTCGCACTACTCT-3'; and glyceraldehyde 3-phosphate dehydrogenase (GAPDH) forward, 5'-CAC CCACTCCTCCACCTTTG-3' and reverse, 5'-CCACCA CCCTGTTGCTGTAG-3'. The abundance of SOX3 mRNA was expressed relative to GAPDH mRNA.

Cell culture, lentiviral production and infection. The MG63 and U2OS osteosarcoma cells, and the 293T cells were purchased from the American Type Culture Collection (Rockville, MD, USA) and maintained in a humidified incubator at $37^{\circ} \mathrm{C} / 5 \% \mathrm{CO}_{2}$. The MG63 and 293T cells were grown in DMEM medium with $10 \%$ fetal bovine serum (FBS), while the U2OS cells was grown in RPMI-1640 medium (all from Invitrogen) with $10 \%$ FBS.

SOX3 shRNA (shSOX3) target sequence (CAAGGAGTT AGTTAAATGC) and a scramble shRNA (shNC) was cloned into the lentiviral vector PLKO.1 (Addgene, Cambridge, MA, USA). Lentivirus was produced by transfecting the 293T cells with the shRNA plasmids and packaging plasmids using Lipofectamine 2000 (Invitrogen) according to the manufacturer's instructions. The viral supernatant was collected and filtered at 48-72 $\mathrm{h}$ following transfection. The cells were infected with the viruses in the presence of $8 \mu \mathrm{g} / \mathrm{ml}$ of Polybrene (Sigma, St. Louis, MO, USA). A U2OS stable cell line was established using puromycin (Sigma) selection.

Western blot analysis. Protein was extracted using ice-cold radio immunoprecipitation assay buffer (Beyotime, Shanghai, China). Equal amounts of protein from each sample were electrophoretically resolved with sodium dodecyl sulfatepolyacrylamide gel electrophoresis (SDS-PAGE) gels and transferred onto nitrocellulose membranes (Millipore, Bedford, MA, USA). After blocking with 5\% skim milk for $1 \mathrm{~h}$ at room temperature, the blots were probed with specific primary antibodies at $4^{\circ} \mathrm{C}$ overnight. The protein expression levels were determined by incubating the membranes with horseradish peroxidase-conjugated secondary antibody (Beyotime) and enhanced chemiluminescence reagent (Millipore). Each sample was examined in triplicate and the protein expression levels were expressed relative to GAPDH. Antibodies used included anti-SOX3 (Abcam, Cambridge, MA, USA; ab183606; 1:500), anti-cyclin D1 (Abcam; ab16663; 1:200), anti-Bax (Abcam; ab32503; 1:2,000), antiBcl-2 (Abcam; ab692; 1:500), anti-Twist (Abcam; ab50581; 1:2,000), anti-matrix metalloproteinase-9 (MMP-9) (Abcam; ab119906; 1:500), anti-GAPDH (Cell Signaling Technology, Danvers, MA, USA; \#5174; 1:1,500), anti-Cdc25A (Cell Signaling Technology; \#3652; 1:1,000), anti-proliferating cell nuclear antigen (PCNA) (Cell Signaling Technology; \#13110; 1:1,000), anti-Snail (Cell Signaling Technology; \#3879S; 1:1,000) and anti-E-cadherin (Cell Signaling Technology;
\#14472; 1:1,000). Goat anti-mouse (A0216; 1:1,000) and goat anti-rabbit (A0208; 1:1,000) secondary antibody (both from Beyotime). The protein expression levels were determined by incubating the membranes with horseradish peroxidase conjugated secondary antibody (Beyotime) at room temperature for $1 \mathrm{~h}$. Enhanced chemiluminescence reagent (Millipore) was applied to examine the protein expression. Western blot analysis was repeated three times and quantification of the blots was performed by using ImageJ software (NIH, Bethesda, MD, USA) with GAPDH as loading control.

Cell proliferation assay. Cell proliferation was examined using the Cell Counting kit-8 CCK-8; Beyotime) following the manufacturer's instructions. Briefly, the MG63 or U2OS cells were plated in 96 -well plates $\left(3 \times 10^{3}\right.$ cells/well). Following overnight incubation, the cells were infected with shNC or shSOX3 lentivirus. Cell proliferation was determined every $24 \mathrm{~h}$ with CCK-8 solution. Each experiment was performed in triplicate.

Tumor xenograft model. All animal experiments were approved by the Ethics Committee of the Department of Orthopedic Surgery, Shanghai Sixth People's Hospital. A total of 12 female athymic nude mice (4 to 5 weeks old, 15-20 g) (SLAC Animal, Shanghai, China) was used in this study. U2OS stable cells $\left(2 \times 10^{6}\right)$ were injected subcutaneously into the left flanks (six mice were injected with shNC-transfected cells and six mice were injected with shSOX3-transfected cells. Tumors were measured every 3 days using a Vernier caliper, and tumor volumes were calculated using the following formula: $\mathrm{V}=0.5 \mathrm{x}$ (length $\mathrm{x}$ width $^{2}$ ). At 24 days following implantation, all mice were sacrificed, and the tumors were isolated, weighed and protein expression in the tumor samples was examined by western blot analysis.

Cell cycle distribution analysis. At $24 \mathrm{~h}$ following infection, the cells were harvested and washed with phosphate-buffered saline (PBS), followed by fixation with ice-cold $70 \%$ ethanol at $-20^{\circ} \mathrm{C}$ overnight. After washing with PBS, the cells were resuspended in PBS containing $0.05 \mathrm{mg} / \mathrm{ml}$ propidium iodide (PI; Sigma) and $100 \mathrm{U} / \mathrm{ml}$ RNase A in the dark at room temperature for $30 \mathrm{~min}$. Samples were analyzed for DNA content on a flow cytometer (BD Biosciences, San Jose, CA, USA).

Analysis of cell apoptosis. At $24 \mathrm{~h}$ following infection, the cells were harvested and washed with PBS, followed by staining with the Annexin V-fluorescein isothiocyanate (FITC)/PI apoptosis assay kit (Beyotime) as instructed by the manufacturer. Samples were analyzed for cell apoptosis on a flow cytometer. Cells undergoing early and late apoptosis were defined by Annexin $\mathrm{V}^{+} / \mathrm{PI}^{-}$staining and Annexin $\mathrm{V}^{+} / \mathrm{PI}^{+}$staining, respectively.

Cell migration and invasion assays. Cell migration assays were performed in Boyden chambers $(8-\mu \mathrm{m}$ pore size; Corning, Corning, NY, USA). The cells $\left(5 \times 10^{4}\right.$ cells/well) transfected with the shRNAs in serum-free medium were seeded into the upper chamber. Medium with $10 \%$ FBS was added to the lower chamber. Following $24 \mathrm{~h}$ of incubation, the cells in the upper chamber were completely removed with a cotton swab. The cells attached to the bottom of the 

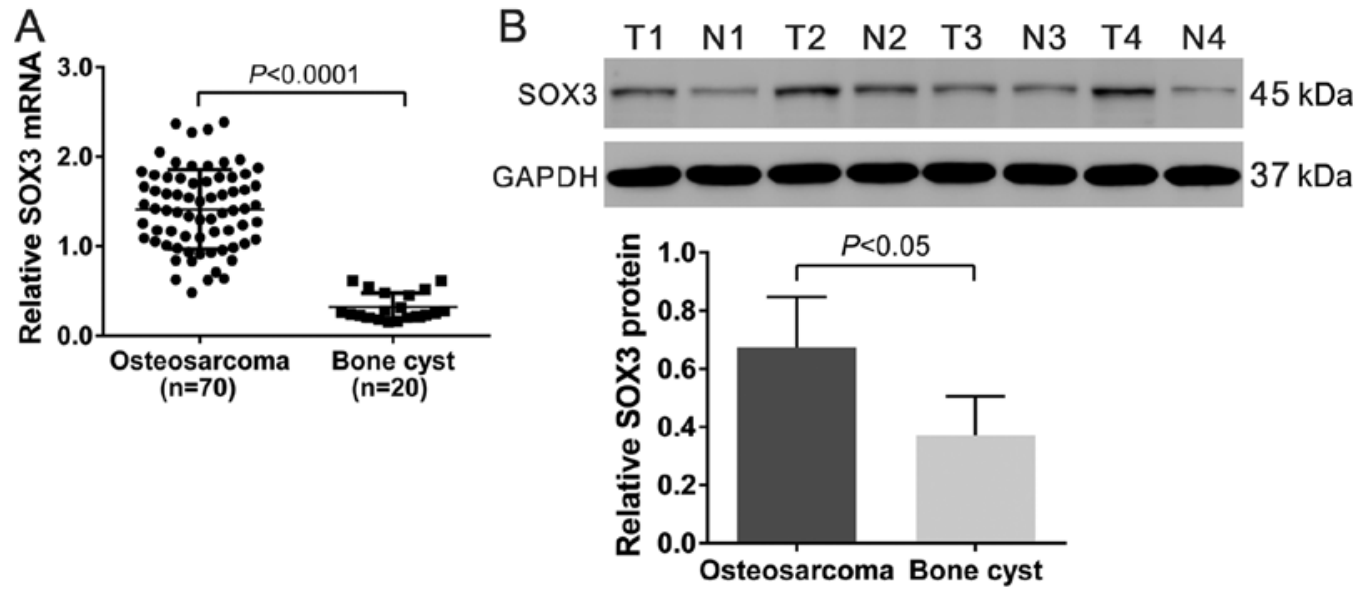

Figure 1. Sex determining region Y-box protein 3 (SOX3) mRNA and protein expression in osteosarcoma and bone cyst tissues. (A) Expression levels of SOX3 mRNA in osteosarcoma tissues $(n=70)$ and non-cancerous bone cyst tissues $(n=20)$ detected by RT-qPCR. (B) Expression levels of SOX3 protein in osteosarcoma tissues and non-cancerous bone tissues by western blot analysis. T1, T2, T3 and T4 refer to osteosarcoma tissues. N1, N2, N3 and N4 refer to noncancerous bone cyst tissues.

A

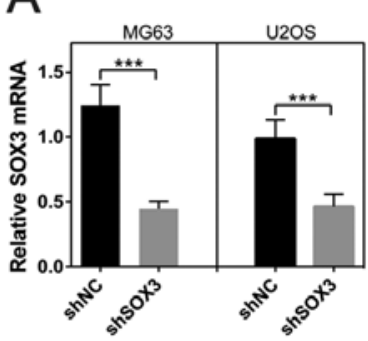

C

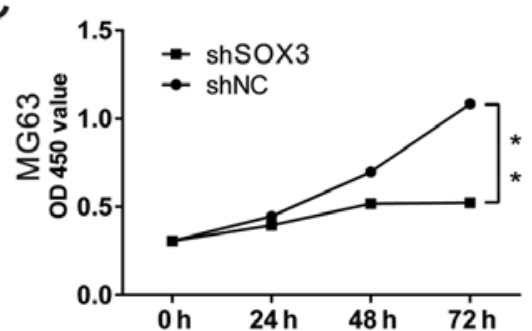

B
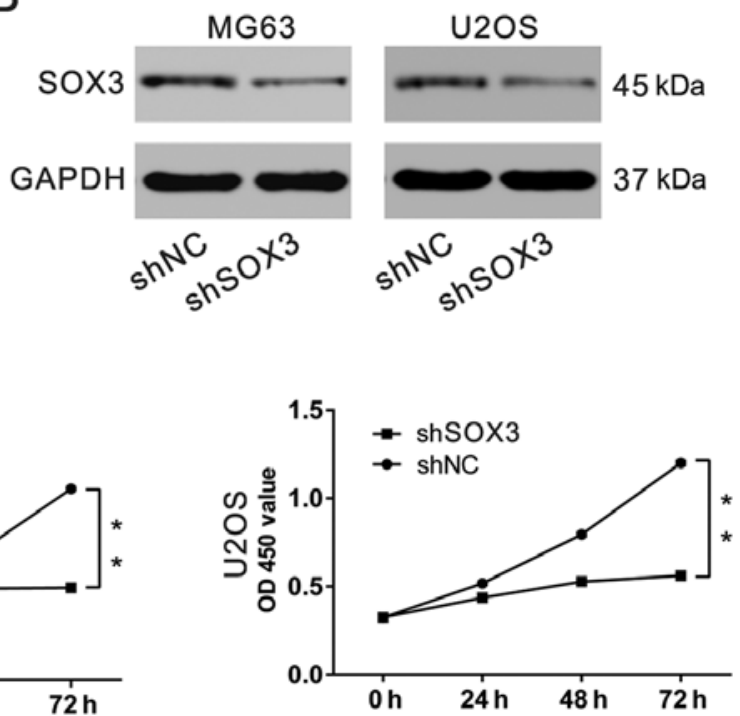

Figure 2. Effects of sex determining region Y-box protein 3 (SOX3) knockdown on the proliferation of osteosarcoma cells in vitro. (A) RT-qPCR and (B) western blot analysis of SOX3 mRNA and protein expression at $48 \mathrm{~h}$ after lentiviral infection of the MG63 and U2OS cells. (C) Cell proliferation after SOX3 knockdown in osteosarcoma cells was measured by cell counting kit-8 (CCK-8) assay at $0,24,48$ and $72 \mathrm{~h}$ after viral infection. The assay was performed in triplicate. ${ }^{* *} \mathrm{P}<0.01$ and ${ }^{* * *} \mathrm{P}<0.001$

membranes were fixed with $4 \%$ paraformaldehyde and stained with $0.5 \%$ crystal violet. The migrated cells were counted in 5 randomly selected fields (x200) under a microscope (Nikon, Tokyo, Japan).

Cell invasion assays were performed in the same manner as the migration assay, with the difference that the upper chamber was pre-coated with $30 \mu 1$ Matrigel (BD Biosciences). Experiments were performed in triplicate.

Statistical analysis. Statistical analysis was carried out using GraphPad Prism software (version 6.0; GraphPad Prism Software, San Diego, CA, USA). The Student's t-test was used for statistical analysis between 2 independent groups. P-values $<0.05$ were considered to indicate statistically significant differences.

\section{Results}

Upregulation of SOX3 in human osteosarcoma tissues. As shown in Fig. 1A, the relative level of SOX3 mRNA in the osteosarcoma tissues $(1.41 \pm 0.05)$ was significantly higher than that in non-cancerous bone cyst tissues $(0.32 \pm 0.03$; $\mathrm{P}<0.0001)$. Similar results were obtained at the protein level (osteosarcoma tissues, 0.67 \pm 0.09 ; bone cyst tissues, 0.37 \pm 0.07 ; $\mathrm{P}<0.05$; Fig. 1B).

SOX3 knockdown inhibits the proliferation of osteosarcoma cells in vitro. To perform thefunctional analysis of SOX3, we knocked down SOX3 expression in two human osteosarcoma cells (MG63 and U2OS) by using a lentivirus system. At $48 \mathrm{~h}$ following infection, the effects of shSOX3 
A

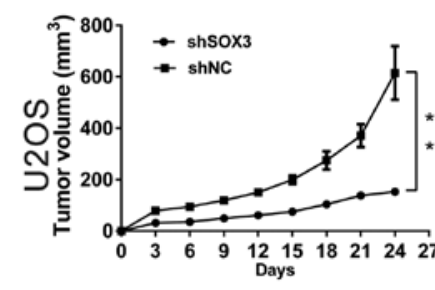

C

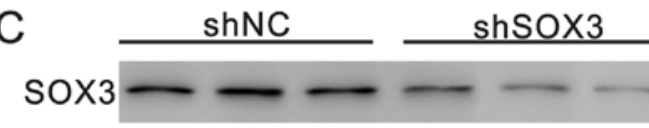

GAPDH
B

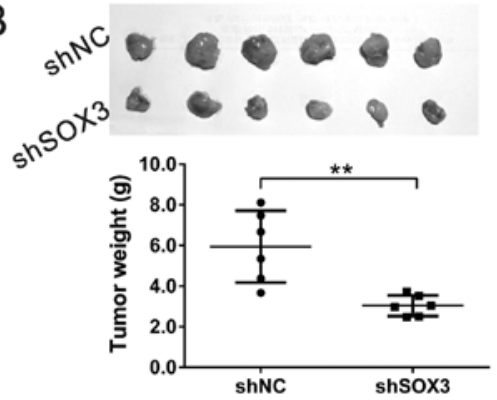

$45 \mathrm{kDa}$

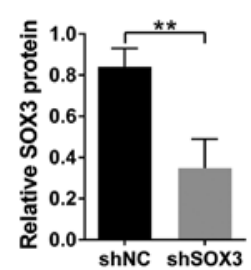

Figure 3. Effects of sex determining region Y-box protein 3 (SOX3) knockdown on the proliferation of U2OS cells in vivo. (A) Growth curve of xenograft tumors derived from U2OS cells stably transfected with SOX3 shRNA (shSOX3) or shNC ( $\mathrm{n}=6)$. (B) After 24 days, xenograft tumors [from mice injected with shNC-transfected cells $(n=6)$ and shSOX3-transfected cells $(n=6)]$ were dissected, photographed (upper panel) and weighed (lower panel). (C) The protein expression of SOS3 in tumor xenografts was assessed by western blot analysis. ${ }^{* *} \mathrm{P}<0.01$.
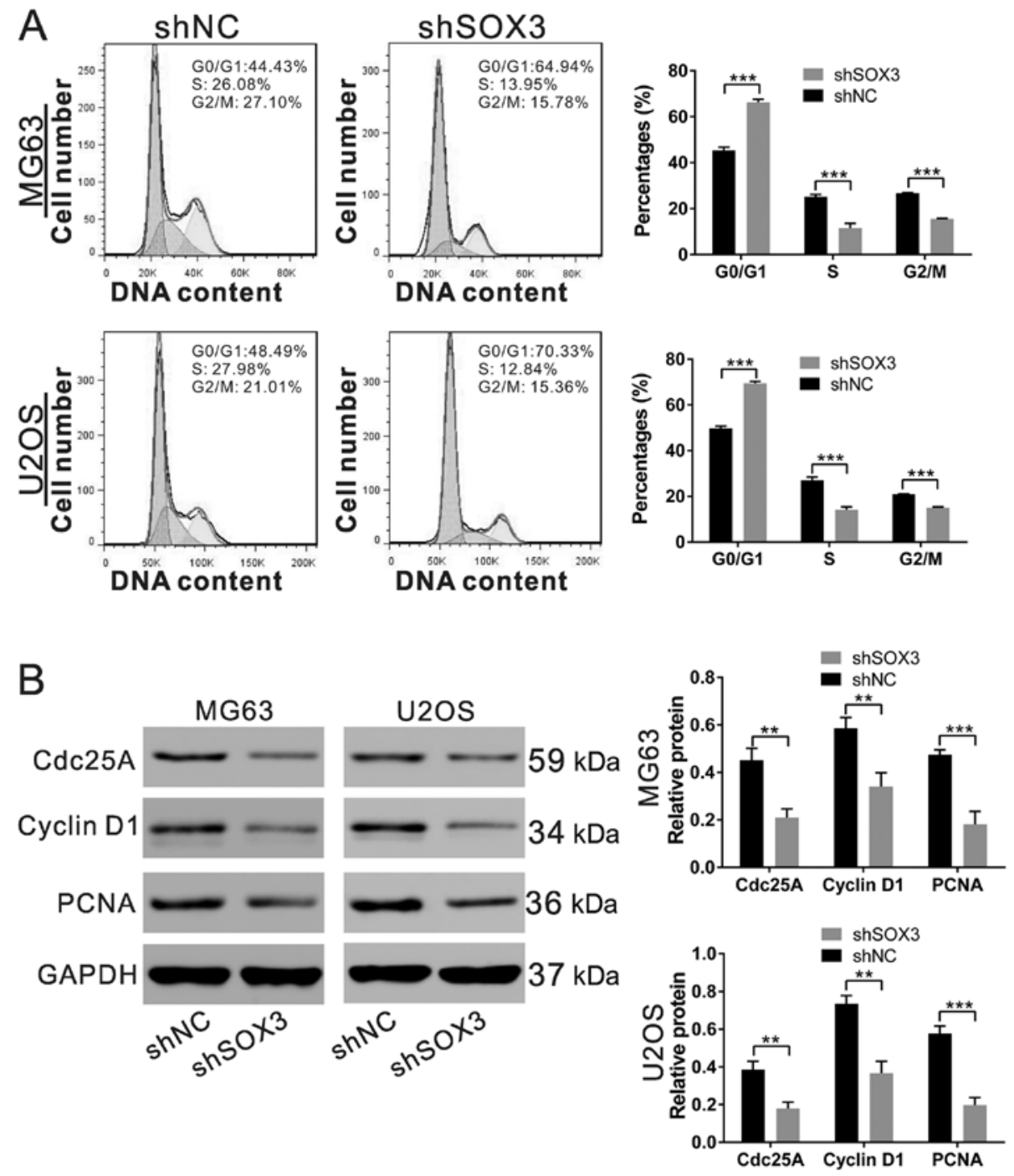

Figure 4. Effects of sex determining region Y-box protein 3 (SOX3) knockdown on cell cycle. (A) Cell cycle analysis of shNC-and SOX3 shRNA (shSOX3)infected cells at $24 \mathrm{~h}$ after infection. DNA content was measured by propidium iodide (PI) staining and flow cytometry analysis. (B) Western blot analysis was performed to compare expression levels of cell-cycle-regulated proteins, Cdc25A, cyclin D1 and PCNA between shNC-and shSOX3-infected cells. ${ }^{* *} \mathrm{P}<0.01$ and ${ }^{* * * *} \mathrm{P}<0.001$. 

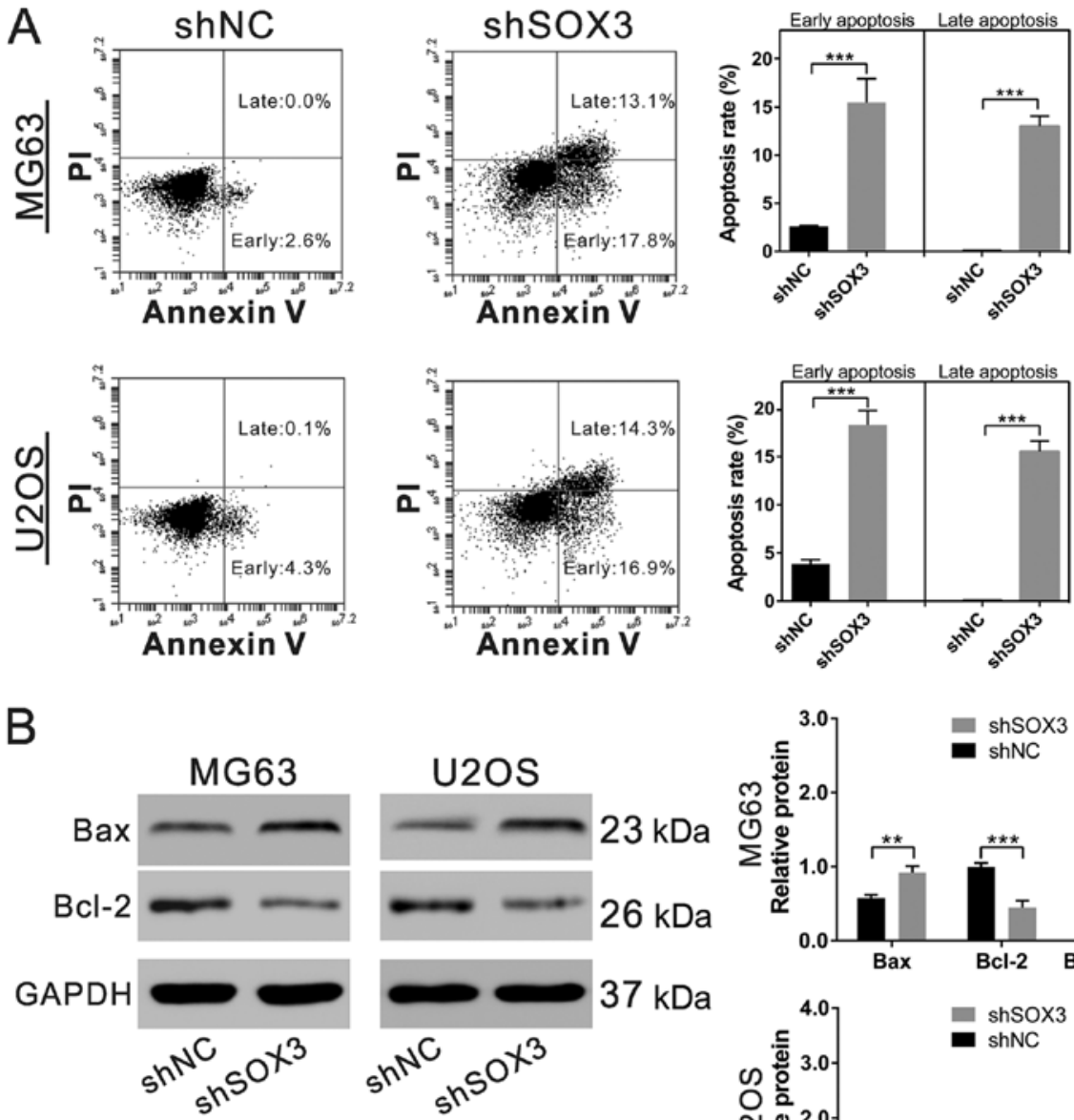

$23 \mathrm{kDa}$

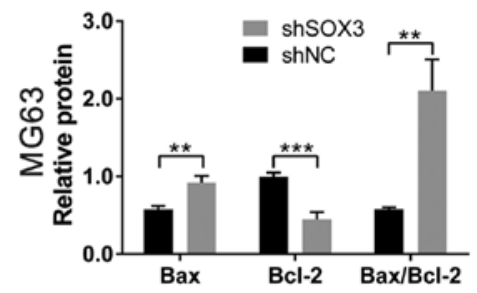

$37 \mathrm{kDa}$

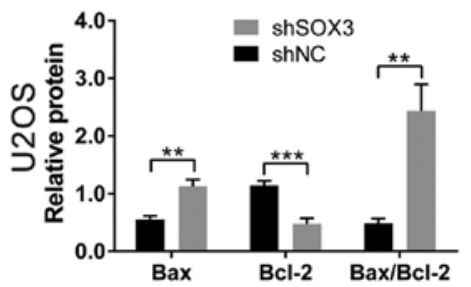

Figure 5. Effects of sex determining region Y-box protein 3 (SOX3) knockdown on cell apoptosis. (A) Cell apoptosis of shNC-and SOX3 shRNA (shSOX3)infected cells at $24 \mathrm{~h}$ after infection was determined by Annexin V/propidium iodide (PI) staining and flow cytometry analysis. (B) Western blot analysis was performed to compare expression levels of cell apoptosis-related proteins, Bax and Bcl-2 between shNC-and shSOX3-infected cells. ${ }^{* *} \mathrm{P}<0.01$ and ${ }^{* * * *} \mathrm{P}<0.001$.

on endogenous SOX3 expression were evaluated. As shown in Fig. 2A and B, SOX3 mRNA and protein expression was markedly inhibited due to shSOX3 infection in both osteosarcoma cells $(\mathrm{P}<0.001)$.

To determine the proliferation rates following the downregulation of SOX3 in the osteosarcoma cell lines, CCK-8 assays were performed over a 3 -day period. We found that both cell lines in which SOX3 was silenced exhibited significant growth inhibition compared with the shNC-infected cells $(\mathrm{P}<0.01$; Fig. 2C).

SOX3 knockdown suppresses tumor growth in a xenograft mouse model. To investigate whether SOX3 knockdown in osteosarcoma cells suppresses tumor growth in vivo, U2OS cells stably transfected with shSOX3 or shNC were subcutaneously implanted into nude mice. As shown in Fig. 3A, the growth rates of xenografts formed from shSOX3 stably transfected cells were much slower than those from shNC cells $(\mathrm{P}<0.01)$. After 24 days, the volumes and weights of the tumors formed from shSOX3-transfefed cells were significantly decreased compared with those of tumors derived from shNC-transfected cells $(\mathrm{P}<0.01$; Fig. 3B). Moreover, SOX3 protein expression decreased by $58.5 \%$ in the xenografts formed from shSOX3-transfected cells, as compared to the xenografts formed from shNC-transfected cells $(\mathrm{P}<0.01$; Fig. 3C). These data suggested that the knockdown of SOX3 inhibited tumor growth in nude mice.

SOX3 knockdown induces G1 phase arrest of osteosarcoma cells. To clarify the mechanisms underlying the growth inhibitory effects of the knockdown of SOX3 by shRNA on osteosarcoma cell lines, cell cycle distribution was analyzed by flow cytometry in the cells stained with PI (Fig. 4A). At $24 \mathrm{~h}$ following infection, a higher proportion of shSOX3-infected cells was observed in the G1 phase (MG63, $66.34 \pm 1.29 \%$; U2OS, $49.68 \pm 1.04 \%$; $\mathrm{P}<0.001)$ in comparison with that of shNC-infected cells (MG63, 45.40 $1.39 \%$; U2OS, $69.52 \pm 0.72 \%$ ). Concomitant decreases were observed in the proportions of cells in $\mathrm{S}$ and $\mathrm{G} 2 / \mathrm{M}$ phases.

To explore the potential molecular mechanisms responsible for shSOX3-induced G1 arrest in osteosarcoma cells, we explored the effects of shSOX3 on the expression of cell cycleregulated proteins. Western blot analysis revealed that the protein levels of Cdc25A, cyclin D1 and PCNA were significantly decreased in the shSOX3-infected cells, compared with the shNC-infected cells (Fig. 4B). 
A

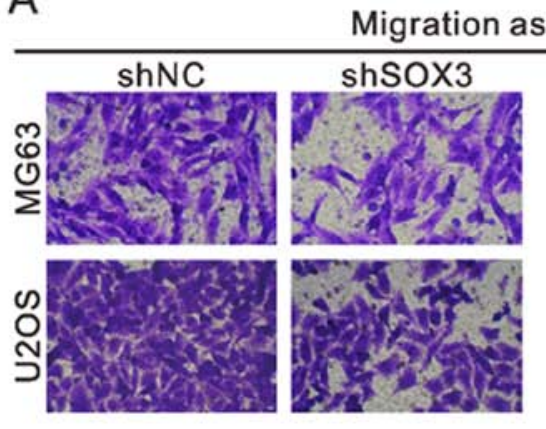

B

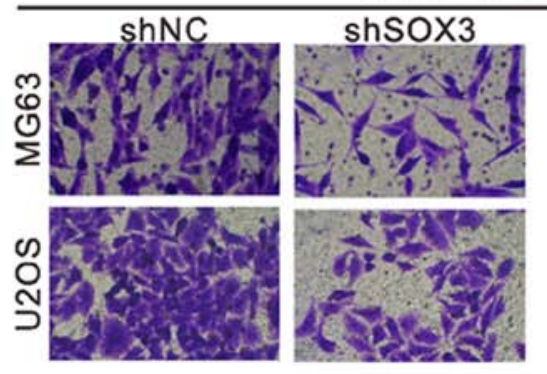

Invasion assay

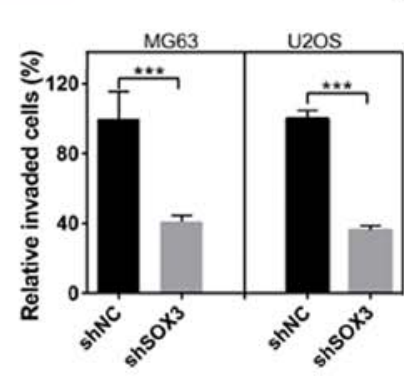

C
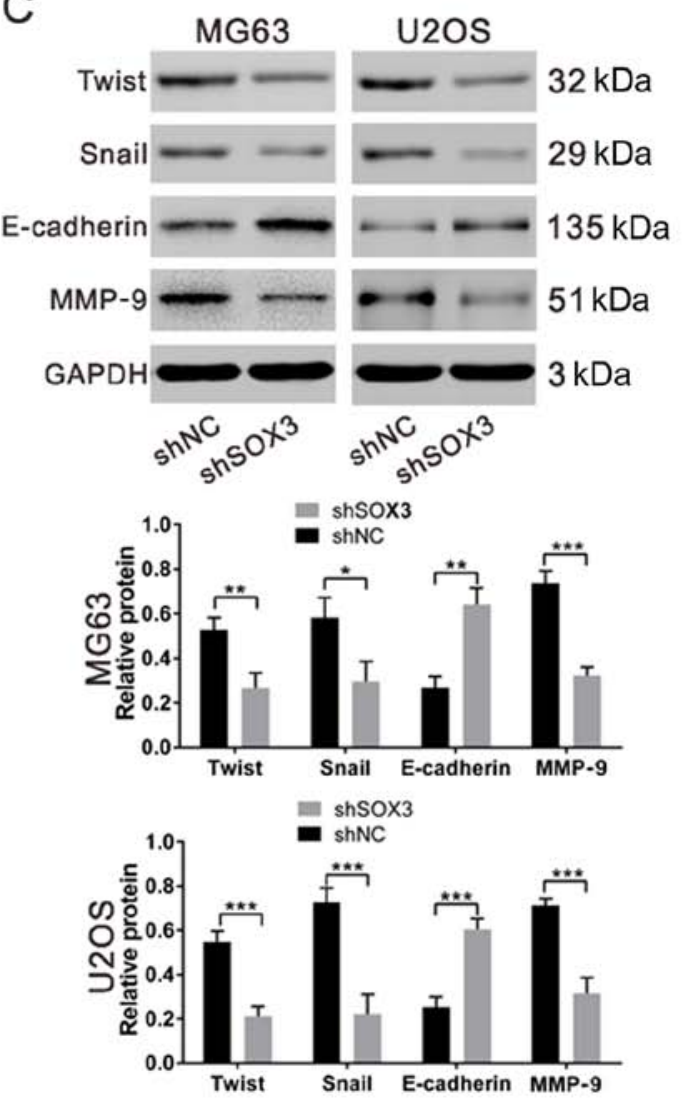

Figure 6. Effects of sex determining region Y-box protein 3 (SOX3) knockdown on cell migration and invasion. (A and B) MG63 or U2OS cells infected with either shNC or SOX3 shRNA (shSOX3) lentivirus were subjected to (A) cell migration and (B) cell invasion assay. The migration or invasion of cells transfected with shNC was set as $100 \%$. (C) Western blot analysis was performed to compare expression levels of epithelial-mesenchymal transition (EMT)-related proteins, Twist, Snail, E-cadherin and MMP-9 between shNC-and shSOX3-infected cells. ${ }^{*} \mathrm{P}<0.05,{ }^{* *} \mathrm{P}<0.01$ and ${ }^{* * *} \mathrm{P}<0.001$.

SOX3 knockdown induces the apoptosis of osteosarcoma cells. To determine whether apoptosis occurred in shSOX3-infected cells, we performed flow cytometry analysis on the cells stained with Annexin V/PI. As shown in Fig. 5A, a significant increase in the percentages of cells undergoing apoptosis was observed in the MG63 cells in which SOX2 was knocked down $(15.50 \pm 2.46$ and $13.00 \pm 1.05 \%$ for early and late apoptosis, respectively) compared with the shNC-infected cells $(2.60 \pm 0.10$ and $0.03 \pm 0.06 \%$ for early and late apoptosis, respectively). Similar results were obtained in the U2OS cells.

To explore the possible molecular mechanisms responsible for the shSOX3-induced apoptosis of osteosarcoma cells, we examined the effects of the silencing of SOX3 by shSOX3 on the expression of cell apoptosis-related proteins. Western blot analysis revealed that the protein levels of Bax, a pro-apoptotic protein (15), were significantly increased in the shSOX3-infected cells, compared with the shNC-infected cells, whereas the expression of $\mathrm{Bcl}-2$, an anti-apoptotic protein (15), was decreased in the shSOX3-infected cells compared with the control cells (Fig. 5B).

SOX3 knockdown suppresses the migration and invasion of osteosarcoma cells. To determine whether SOX3 affects the migration and invasion of osteosarcoma cells, Transwell assay was performed (Fig. 6A). The shSOX3-infected cells (both cell lines) exhibited a significant decrease in migration and invasion compared with the shNC-infected cells.
It has been noted that epithelial-mesenchymal transition (EMT) is a critical event during tumor invasion and metastasis (16). The effect of SOX3 knockdown on the expression of EMT-related proteins was also explored. The protein levels of EMT-promoting proteins [Twist, Snail and MMP-9 (17)] were significantly decreased in the cells in which SOX3 was knocked down, whereas the expression of the main factor of EMT [E-cadherin (17)] was markedly elevated in comparison with the shNC-infected cells.

\section{Discussion}

Previous studies have reported that SOX3 expression is often increased in human esophageal squamous cell carcinoma (ESCC) (18) and epithelial ovarian cancer (9). In the current study, we found that SOX3 expression was upregulated in osteosarcoma, suggesting the oncogenic role of SOX3. Furthermore, we performed the functional characterization of SOX3 in osteosarcoma cell lines by lentivirus-mediated RNA interference. Previous studies have suggested the promoting effect of SOX3 on the proliferation of ESCC (10) and epithelial ovarian cancer cell lines (9). In this study, we found that the silencing of SOX3 inhibited cell proliferation, migration and invasion. SOX3 plays an oncogenic role and may be a potential target for osteosarcoma treatment.

In general, deregulated cell cycle and apoptosis are main causes for uncontrolled proliferation (19). Several members 
of SOX family proteins, including SOX2 (20), SOX7 (21) and SOX21 (22), have been shown to regulate cell cycle and apoptosis in diverse cell lines. In this study, flow cytometric analysis revealed that SOX3 knockdown in osteosarcoma cells induced G1 phase arrest and apoptosis. We demonstrated that decreased protein levels of Cdc25A, cyclin D1, PCNA and Bcl-2, and an increased protein level of Bax were associated with SOX3 knockdown. These results were consistent with the findings of G1 phase arrest and increased cell apoptosis observed in the shSOX3-infected osteosarcoma cells. It has been reported that Src (23) activates the transcription of cyclin D1. Cyclin D1 (24) and $\mathrm{Cdc} 25 \mathrm{~A}(25)$ are direct $\mathrm{TCF} / \beta$-catenin transcriptional targets. SOX3 targets Src kinase in epithelial ovarian cancer cell lines (9), whereas the overexpression of Xenopus SOX3 was found to inhibit $\beta$-catenin activity $(26,27)$. Thus, further studies are warranted to whether SOX3 functions in the cell cycle through Src or Wnt signals in osteosarcoma cells.

We further elucidated that SOX3 knockdown suppressed cell migration and invasion. We demonstrated that SOX3 knockdown led to decreased protein levels of Twist, Snail and MMP-9, and increased protein level of E-cadherin. It has been reported that other SOX family members, such as SOX2 and SOX4, promote cell migration and invasion in breast, prostate and liver cancer cells via regulating EMT (28-31). SOX family members have been shown to regulate the expression of EMT-related protein, including Snail, ZEB1, Twist and E-cadherin $(28,32,33)$, being consistent with our finding. In chicken embryo, SOX3 is found to repress Snail expression (7). The discrepancy between this finding and our data may be due to different species and biology processes. Further studies are required to clarify the mechanism how SOX3 regulates the expression of EMT-related proteins.

In conclusion, we revealed that SOX3 expression was frequently upregulated in osteosarcoma. Moreover, we also showed that silencing of SOX3 expression inhibited cell proliferation through cell cycle arrest and apoptosis in osteosarcoma cells, as well as suppressed cell migration and invasion. To the best of our knowledge, we have demonstrated, for the first time, SOX3 acts as an oncogene in osteosarcoma, and SOX3 inhibitors or downstream effectors may be interesting targets for osteosarcoma therapy.

\section{Acknowledgements}

Not applicable.

\section{Funding}

No funding was received.

\section{Availability of data and materials}

The analyzed data sets generated during the study are available from the corresponding author on reasonable request.

\section{Authors' contributions}

YG and XY conceived and designed the study. YG, JY and MT performed the experiments. YG, JY and XY wrote the manuscript. All authors read and approved the manuscript.

\section{Ethics approval and consent to participate}

For the use of human sample, approval was obtained by the Ethics Committees of Shanghai Sixth People's Hospital. Written consent was obtained from all enrolled patients for the use of tissue specimens. All animal experiments were approved by the Ethics Committee of Department of Orthopedic Surgery, Shanghai Sixth People's Hospital.

\section{Consent for publication}

Not applicable.

\section{Competing interests}

The authors declare that they have no competing interests.

\section{References}

1. Rabinowicz R, Barchana M, Liphshiz I, Futerman B, Linn S and Weyl-Ben-Arush M: Cancer incidence and survival among children and adolescents in Israel during the years 1998 to 2007. J Pediatr Hematol Oncol 34: 421-429, 2012.

2. Mirabello L, Troisi RJ and Savage SA: Osteosarcoma incidence and survival rates from 1973 to 2004: Data from the Surveillance, Epidemiology, and End Results Program. Cancer 115: 1531-1543, 2009.

3. Ferguson WS and Goorin AM: Current treatment of osteosarcoma. Cancer Invest 19: 292-315, 2001.

4. Sarkar A and Hochedlinger K: The sox family of transcription factors: Versatile regulators of stem and progenitor cell fate. Cell Stem Cell 12: 15-30, 2013.

5. Alatzoglou KS, Azriyanti A, Rogers N, Ryan F, Curry N, Noakes C, Bignell P, Hall GW, Littooij AS, Saunders D, et al: SOX3 deletion in mouse and human is associated with persistence of the craniopharyngeal canal. J Clin Endocrinol Metab 99: E2702-E2708, 2014.

6. Archer TC, Jin J and Casey ES: Interaction of Sox1, Sox2, Sox3 and Oct4 during primary neurogenesis. Dev Biol 350: 429-440, 2011.

7. Acloque H, Ocaña $\mathrm{OH}$, Matheu A, Rizzoti K, Wise C, Lovell-Badge R and Nieto MA: Reciprocal repression between Sox 3 and snail transcription factors defines embryonic territories at gastrulation. Dev Cell 21: 546-558, 2011.

8. Xia Y, Papalopulu N, Vogt PK and Li J: The oncogenic potential of the high mobility group box protein Sox3. Cancer Res 60: 6303-6306, 2000.

9. Yang J, Yuan D, Li J, Zheng S and Wang B: miR-186 downregulates protein phosphatase PPM1B in bladder cancer and mediates G1-S phase transition. Tumour Biol 37: 4331-4341, 2016.

10. Cai QY, Liang GY, Zheng YF, Tan QY, Wang RW and Li K: Sox3 silencing inhibits metastasis and growth of esophageal squamous cell carcinoma cell via Downregulating GSK-3 $\beta$. Int J Clin Exp Pathol 9: 2939-2949, 2016.

11. Basu-Roy U, Seo E, Ramanathapuram L, Rapp TB, Perry JA, Orkin SH, Mansukhani A and Basilico C: Sox2 maintains self renewal of tumor-initiating cells in osteosarcomas. Oncogene 31: 2270-2282, 2012 .

12. Yang C, Hou C, Zhang H, Wang D, Ma Y, Zhang Y, Xu X, Bi Z and Geng S: miR-126 functions as a tumor suppressor in osteosarcoma by targeting Sox2. Int J Mol Sci 15: 423-437, 2013.

13. Zhu H, Tang J, Tang M and Cai H: Upregulation of SOX9 in osteosarcoma and its association with tumor progression and patients' prognosis. Diagn Pathol 8: 183, 2013.

14. Wu Z, Liu J, Wang J and Zhang F: SOX18 knockdown suppresses the proliferation and metastasis, and induces the apoptosis of osteosarcoma cells. Mol Med Rep 13: 497-504, 2016.

15. Oltval ZN, Milliman CL and Korsmeyer SJ: Bcl-2 heterodimerizes in vivo with a conserved homolog, Bax, that accelerates programed cell death. Cell 74: 609-619, 1993.

16. Yang J and Weinberg RA: Epithelial-mesenchymal transition: At the crossroads of development and tumor metastasis. Dev Cell 14: 818-829, 2008. 
17. Huber MA, Kraut $\mathrm{N}$ and Beug $\mathrm{H}$ : Molecular requirements for epithelial-mesenchymal transition during tumor progression. Curr Opin Cell Biol 17: 548-558, 2005.

18. Li K, Wang RW, Jiang YG, Zou YB and Guo W: Overexpression of Sox 3 is associated with diminished prognosis in esophageal squamous cell carcinoma. Ann Surg Oncol 20 (Suppl 3): S459-S466, 2013.

19. Evan GI and Vousden KH: Proliferation, cell cycle and apoptosis in cancer. Nature 411: 342-348, 2001.

20. Otsubo T, Akiyama Y, Yanagihara K and Yuasa Y: SOX2 is frequently downregulated in gastric cancers and inhibits cell growth through cell-cycle arrest and apoptosis. Br J Cancer 98: 824-831, 2008.

21. Zhang Y, Huang S, Dong W, Li L, Feng Y, Pan L, Han Z, Wang X, Ren G, Su D, et al: SOX7, downregulated in colorectal cancer, induces apoptosis and inhibits proliferation of colorectal cancer cells. Cancer Lett 277: 29-37, 2009.

22. Ferletta M, Caglayan D, Mokvist L, Jiang Y, Kastemar M, Uhrbom L and Westermark B: Forced expression of Sox 21 inhibits Sox 2 and induces apoptosis in human glioma cells. Int J Cancer 129: 45-60, 2011.

23. Lee RJ, Albanese C, Stenger RJ, Watanabe G, Inghirami G, Haines GK III, Webster M, Muller WJ, Brugge JS, Davis RJ, et al: $\mathrm{pp} 60(\mathrm{v}-\mathrm{src})$ induction of cyclin D1 requires collaborative interactions between the extracellular signal-regulated kinase, p38, and Jun kinase pathways. A role for cAMP response element-binding protein and activating transcription factor-2 in pp60(v-src) signaling in breast cancer cells. J Biol Chem 274 7341-7350, 1999.

24. Shtutman M, Zhurinsky J, Simcha I, Albanese C, D'Amico M, Pestell R and Ben-Ze'ev A: The cyclin D1 gene is a target of the beta-catenin/LEF-1 pathway. Proc Natl Acad Sci USA 96 5522-5527, 1999.

25. Vijayakumar S, Liu G, Rus IA, Yao S, Chen Y, Akiri G, Grumolato L and Aaronson SA: High-frequency canonical Wnt activation in multiple sarcoma subtypes drives proliferation through a TCF/ $\beta$-catenin target gene, CDC25A. Cancer Cell 19: 601-612, 2011
26. Kormish JD, Sinner D and Zorn AM: Interactions between SOX factors and $\mathrm{Wnt} / \beta$-catenin signaling in development and disease. Dev Dyn 239: 56-68, 2010.

27. Zorn AM,Barish GD, Williams BO,Lavender P,Klymkowsky MW and Varmus HE: Regulation of Wnt signaling by Sox proteins: XSox 17 alpha/beta and XSox 3 physically interact with betacatenin. Mol Cell 4: 487-498, 1999.

28. Li X, Xu Y, Chen Y, Chen S, Jia X, Sun T, Liu Y,Li X, Xiang R and LiN: SOX2 promotes tumor metastasis by stimulating epithelial-tomesenchymal transition via regulation of $\mathrm{WNT} / \beta$-catenin signal network. Cancer Lett 336: 379-389, 2013.

29. Yang N, Hui L, Wang Y, Yang H and Jiang X: SOX2 promotes the migration and invasion of laryngeal cancer cells by induction of MMP-2 via the PI3K/Akt/mTOR pathway. Oncol Rep 31: 2651-2659, 2014

30. Yang N, Hui L, Wang Y, Yang H and Jiang X: Overexpression of SOX2 promotes migration, invasion, and epithelial-mesenchymal transition through the $\mathrm{Wnt} / \beta$-catenin pathway in laryngeal cancer Hep-2 cells. Tumour Biol 35: 7965-7973, 2014.

31. Jafarnejad SM, Wani AA, Martinka M and Li G: Prognostic significance of Sox 4 expression in human cutaneous melanoma and its role in cell migration and invasion. Am J Pathol 177: 2741-2752, 2010.

32. Parvani JG and Schiemann WP: Sox4, EMT programs, and the metastatic progression of breast cancers: Mastering the masters of EMT. Breast Cancer Res 15: R72, 2013.

33. Guan Z, Zhang J, Wang J, Wang H, Zheng F, Peng J, Xu Y, Yan M, Liu B, Cui B, et al: SOX1 Downregulates $\beta$-catenin and reverses malignant phenotype in nasopharyngeal carcinoma. Mol Cancer 13: 257, 2014. 